\title{
Research on the Model for Route Selection of Railway Pickup and Delivery Service
}

\author{
Weizhe Wang ${ }^{1, ~ a, ~ X i a o d o n g ~ Z h a n g ~}{ }^{1, b}$, Maoxiang Lang ${ }^{1, c, ~}{ }^{*}$, Xueqiao $\mathrm{Yu}^{1, \mathrm{~d}}$ and \\ Xiao $\mathrm{Yu}^{2, \mathrm{e}}$
}

${ }^{1}$ School of Traffic and Transportation, Beijing Jiaotong University, Beijing, 100044, China.

2Signal and Communication Research Institute, China Academy of Railway Sciences, Beijing, 100081, China.

a16120890@bjtu.edu.cn, bzhangxd@bjtu.edu.cn, c, ${ }^{*}$ mxlang@bjtu.edu.cn, dyuxueqiao@bjtu.edu.cn, eluck1036@163.com

\begin{abstract}
With economic expansion having moderated to a "new normal" pace, the railway freight market share continues to be sluggish, and China's railways are transforming into modern logistics and carrying out pickup and delivery services. This paper mainly focuses on the transportation lines of the external operations of the station. It is fully analyzing that the transferring of the railway is highway transportation for the connection between the customer and the station. According to the mixed integer linear programming model with 0-1 variables based on comprehensive cost optimization, this paper built the model of pickup and delivery route selection, to determine the running route of the vehicle during the service process, as well as make sure the requirements of each customer can be satisfied and the total cost is considering. LING012.0 is used to calculate the optimal solution, and we optimized the railway pickup and delivery service route. Finally, the model is validated by the data of one day's bulk cargo pickup and delivery at a railway station of Shanghai as an example.
\end{abstract}

Keywords: Pickup and delivery; Railway; Route optimization; 0-1 mixed integer programming.

\section{Introduction}

With the of the development of modern industry and the diversification of customer demand, pickup and delivery service for railway door-to-door transport has become the inevitable direction of the future development of Railway Logistics in China. In 2015, General Manager Sheng Guangzu proposed six goals to promote the modern logistics construction of the railway at the meeting of the railway modern logistics construction. The third point is to fully implement the delivery service and strengthen the delivery service, improving the delivery capacity and cargo handling level for the "last mile" of railway freight services. The key to pickup and delivery is how to scientifically and reasonably match the relationship between capacity, transportation network and demand. The essence is the pickup and delivery routing problem (or Vehicle Routing Problem), based on this, a model was built and studied. Because the network that involved in this problem is large, it is not appropriate to adopt an accurate solution. The heuristic algorithm is mainly used to solve the VRP problem, but the optimal solution cannot be obtained. Considering present situation, China's pickup and delivery service is still developing, meanwhile the total amount is low, and railways account for a relatively small proportion of the national freight market, the portion with door-to-door demand is ever smaller. The pickup and delivery service directly handled by freight stations, the scope of services is limited. So, the case network is limited in size, and an accurate solution strategy can be considered.

\section{Research on Route Selection Model for Pickup and Delivery Service}

\subsection{The Essence of the Route for Pickup and Delivery Service}

The transportation line is the channel that realizes the spatial displacement of people or objects, and it is an important basis for transportation. The pick-up and delivery route refer to the operation channel for carrying the operator and the carrier to complete the pick-up and delivery work. It is mainly based on roads and railways in the process of railway pick-up and delivery. According to the 
way of pickup and delivery, the composition of transportation lines is also different. For the customer with special railway lines, door-to-door transport can be realized directly by railway. The goods are directly transporting to the customer's factory and warehouse by special railway lines. In this case, the pickup and delivery routes are mainly composed of railway special lines; For customers who do not have special railway lines, in order to pickup and delivery, the customer and the freight station must be connected by road transportation. In this case, the way of pick-up and delivery refers to road transportation. This paper mainly focuses on the customers who don't have special railway lines.

\subsection{The Classification of Pickup and Delivery Route Based on Operational Process}

According to the operation process, the pickup and delivery route can be divided into two parts: the internal transporting route of the station and the external transporting route of the station. The internal transporting route include the routes for loading and unloading goods, and for the operators and the operating facilities (such as Forklift truck and gantry crane, etc.) during the vehicle leaving the station, and the routes for the vehicles which used for pickup and delivery. When designing this part of the transport route, we should fully consider the safety of the operation to ensure the efficiency of transport, trying to avoid the crossing and conflict of streamlines. In particular, people and vehicles should be separated as far as possible. The external transporting route of the station mainly refers to the driving route from the station to the door to pick up and delivery. and those for mainly refers to the running route of the vehicle from the yard station to the door to fetch goods and distribute goods. Due to the randomness of customer demand, the driving route of pickup and delivery in each operation is quite different. Especially when the number of customers in a single operation is large, how to allocate the capacity scientifically and reasonably, and reduce the cost of the operation and improve the operation efficiency as much as possible to meet the needs of all customers, has become an important direction of research. The expressions referred to in the following paragraphs for the routes of pick-up and delivery refer to the "external transporting route".

\subsection{General Idea}

The core of the pick-up and delivery route in this paper is to determine the driving route of the vehicle during the service process, so that the requirements of each customer can be satisfied and the total transportation cost is the lowest. The essence is to determine the service order of each of the demand points (customers) that are attached to the vehicles of the station. This paper adopts the modeling idea of Vehicle Routing Problem (VRP), and the LINGO software is used to optimize the solution to obtain the optimal solution.

\section{The Establishment and Solution of the Mathematical Model of Transportation Routes Considering Pickup and Delivery at the Same Time}

\subsection{Problem Description}

A railway station has a number of vehicles available for pickup and delivery. The vehicle's capacity is certain. The station needs to meet the needs of several customers in a certain scheduling. The delivery requirements of each customer are all known, and the transportation cost between each node is known. All vehicles depart from the station, bring the goods to the customers who need to deliver the goods, and take the goods of the customers who need to pick up the goods, and finally return to the original station. When the vehicle load is limited and cannot continue to work, the vehicle returns to the station, and the other vehicles continue to service the remaining customers until all customer needs are met. The purpose of the model is to get a vehicle scheduling scheme for the lowest operating cost of the vehicle under various restrictions. There are several basic hypotheses in this study:

(1) With the sufficient delivery capacity, a single vehicle can only complete one line of service.

(2) All vehicles depart from the station and return to the original station.

(3) Vehicle load is known and identical, no overload is allowed, and the rate is the same.

(4) The vehicle must meet the delivery requirements of each customer, and the demand is known. 
(5) The goods cannot be disassembled. The pickup service or delivery service of the same customer can only be done by one car, but the pick-up and delivery of the goods can be done by two different vehicles, that is, the demand of a single customer can't exceed the load of the vehicle.

(6) The goods of different cargo owners can be installed in the same vehicle.

(7) The distance between each cargo owner and each station is known.

(8) Regardless of the road conditions, that means the vehicle always maintains a constant speed.

\subsection{Variable Declaration and Model Establishment}

A railway station for pick-up and delivery has a number of vehicles available for pick-up and delivery. The vehicle's capacity is certain. The station needs to meet the needs of several customers in a certain scheduling. The delivery requirements of each customer are all known, and the transportation cost between each node is known. All vehicles depart from the station, bring the goods to the customers who need to deliver the goods, and take the goods of the customers who need to pick up the goods, and finally return to the original station. When the vehicle load is limited and cannot continue to work, the vehicle returns to the station, and the other vehicles continue to service the remaining customers until all customer needs are met. The purpose of the model is to get a vehicle scheduling scheme for the lowest operating cost of the vehicle under various restrictions. This paper mainly considers the optimization of operation cost and efficiency, that is, the optimal scheduling scheme of ton-kilometer and running distance, and establishes a vehicle routing problem model considering both pickup and delivery, as shown below.

$$
\operatorname{Min} Z=\sum_{k=1}^{K} \sum_{j=1}^{N} \sum_{i=1}^{N} c_{i j} y_{i j}^{k}+\sum_{k=1}^{K} \sum_{j=1}^{N} \sum_{i=1}^{N} \operatorname{dist}_{i j} x_{i j}^{k}
$$

s. t.

$$
\begin{gathered}
\sum_{k=1}^{K} \sum_{j=1}^{N} x_{i j}^{k}=1 \quad \forall i \in I i \neq 1 \\
\sum_{j=1}^{N} x_{i j}^{k}=\sum_{j=1}^{N} x_{j i}^{k} \forall k \in K \forall i \in I i \neq 1 \\
y_{i j}^{k} \leq Q x_{i j}^{k} \forall i, j \in I \quad \forall k \in K \\
\sum_{j=2}^{N} y_{1 j}^{k}=\sum_{i=1}^{N} d_{i} \sum_{j=1}^{N} x_{i j}^{k} \forall k \in K \\
\sum_{j=2}^{N} y_{j 1}^{k}=\sum_{i=1}^{N} p_{i} \sum_{j=1}^{N} x_{i j}^{k} \forall k \in K \\
\sum_{i=1, i \neq j}^{N} y_{i j}^{k}+\left(p_{j}-d_{j}\right) \sum_{i=1, i \neq j}^{N} x_{i j}^{k}=\sum_{i=1, i \neq j}^{N} y_{j i}^{k} \forall j \in I j \neq 1 \\
\sum_{i=1}^{N} x_{i 1}^{k}=\sum_{i=1}^{N} x_{1 i}^{k} \forall k \in K \\
x_{i j}^{k}=0 \forall i, j \in I \quad i=j
\end{gathered}
$$




$$
\begin{gathered}
\sum_{i=1}^{N} x_{i 1}^{k} \leq 1 \quad \forall k \in K \\
x_{i j}^{k}=\left\{\begin{array}{l}
0 \\
1
\end{array} \forall i, j \in I \forall k \in K\right. \\
y_{i j}^{k} \geq 0 \quad \forall i, j \in I \forall k \in K
\end{gathered}
$$

$I=\{1,2, \ldots, N\}$ is the set of all nodes, $i, j=1$ is the station, it means each customer when $i, j \neq 1$; $k=\{1,2, \ldots, K\}$ represents the collection of vehicles; $x_{i j}^{k}$ is a $0-1$ variable, it is taken 1 to indicate that the car is driving from the point $\mathrm{i}$ to the point $\mathrm{j}$ is true, otherwise it is false; $y_{i j}^{k}$ indicates the load of the vehicle $\mathrm{k}$ when it is moving from $\mathrm{i}$ to $\mathrm{j} ; p_{i}$ is the pick-up demand of customer $\mathrm{i}$, and $d_{i}$ is the delivery demand of customer I; The vehicle load limit is Q; The transportation cost between nodes $i$ and $j$ is $C_{i j}$, and the distance between nodes $i$ and $j$ is $d_{i j}$.

Explain the objective function and the constraints as follows:

Equation 1 represents the objective function, aiming at the combined minimum of ton-kilometers and the empty driving distance by the dispatched vehicles.

Equation 2 indicates that each customer can and can only be served by one vehicle;

Equation 3 is the vehicle flow balance constraint in customer node;

Equation 4 represents the vehicle limit load constraint

Equations 5,6 and 7 represent the flow balance constraint;

Equation 8 is the vehicle flow balance constraint in station node;

Equation 9 indicates that the vehicle will not driving from the i node to the i node.

Equation 10 indicates that each vehicle can only be scheduled once in all missions;

Equation 11 and 12 defines the decision variables $\mathrm{x}$ and $\mathrm{y}$.

\section{The Case Analysis}

\subsection{Case Introduction}

This paper will verify the validity and feasibility of the model through the case analysis with LINGO12.0. The data of case comes from the statistics of the bulk cargo pickup and delivered on a certain day on a certain railway station in Shanghai.

On that day, the station provided pick-up and delivery services to 10 customers. The 10 customers were located at No. 461, Miyun Road, Shanghai, Xinlian Village, Chongqing, Xinlian Village, No. 286, Zhonghuaxin Road, No. 60, Mudanjiang Road, Yi 4288 Xian Road, Lane 399 Wanrong Road, No. 817 Lingshi Road, No. 681 Dongtang Road, No. 45 Minde Road, No. 1150, Minjiang Road, named as Customer 1, Customer 2,..., Customers 10. The distance between the station and each customer is shown in Table 1. 
Table 1. List of distances between customers

\begin{tabular}{cccccccccccc}
\hline Dist $/ \mathrm{km}$ & station & Cust.1 & Cust.2 & Cust.3 & Cust.4 & Cust.5 & Cust.6 & Cust.7 & Cust.8 & Cust.9 & Cust.10 \\
\hline station & 0 & 5.2 & 37.6 & 7.2 & 21.1 & 12.5 & 4.4 & 4.1 & 15.6 & 6.6 & 6.7 \\
Cust.1 & 5.2 & 0 & 35.6 & 4.8 & 11.3 & 11.1 & 6.2 & 6.8 & 12.5 & 5.7 & 5.7 \\
Cust.2 & 37.6 & 35.6 & 0 & 36.4 & 48.6 & 45.8 & 37 & 35.9 & 51.9 & 36.8 & 37 \\
Cust.3 & 7.2 & 4.8 & 36.4 & 0 & 12.6 & 14.2 & 4.1 & 5.8 & 16.7 & 1.1 & 1.2 \\
Cust.4 & 21.1 & 11.3 & 48.6 & 12.6 & 0 & 27.2 & 17.4 & 20.9 & 17.2 & 12.9 & 13 \\
Cust.5 & 12.5 & 11.1 & 45.8 & 14.2 & 27.2 & 0 & 15.9 & 15.2 & 13.4 & 14.6 & 14.7 \\
Cust.6 & 4.4 & 6.2 & 37 & 4.1 & 17.4 & 15.9 & 0 & 2.4 & 19 & 4.6 & 4.7 \\
Cust.7 & 4.1 & 6.8 & 35.9 & 5.8 & 20.9 & 15.2 & 2.4 & 0 & 18.4 & 6.4 & 6.5 \\
Cust.8 & 15.6 & 12.5 & 51.9 & 16.7 & 17.2 & 13.4 & 19 & 18.4 & 0 & 17.7 & 17.9 \\
Cust.9 & 6.6 & 5.7 & 36.8 & 1.1 & 12.9 & 14.6 & 4.6 & 6.4 & 17.7 & 0 & 0.11 \\
Cust.10 & 6.7 & 5.7 & 37 & 1.2 & 13 & 14.7 & 4.7 & 6.5 & 17.9 & 0.11 & 0 \\
\hline
\end{tabular}

The third-party carrier provides the vehicle to the station for pickup and delivery service, the number of vehicles is sufficient, and the carrying capacity is 8 tons. According to the survey, the average delivery cost per ton-kilometer of bulk cargo is about 6.6 yuan; the specific demand for pickup delivery of each customer is shown in Table 2.

Table 2. List of customer demand information

\begin{tabular}{ccccccccccc}
\hline Demand/T & Cust.1 & Cust. 2 & Cust. 3 & Cust.4 & Cust.5 & Cust.6 & Cust.7 & Cust.8 & Cust.9 & Cust.10 \\
\hline Delivery & 0 & 0 & 0 & 2 & 2.3 & 0.666 & 4.003 & 3.85 & 0.167 & 7.16 \\
Pickup & 0.05 & 0.3 & 0.1 & 0 & 0 & 0 & 0 & 0 & 0 & 0 \\
\hline
\end{tabular}

\subsection{Calculation Results}

We write the model and data to LINGO12.0 and run the solution. The operating environment is windows7, Intel(R)Core TM i7-3632QM CPU@2.20GHZ, and finally get the global optimal solution, as shown in Fig.1.

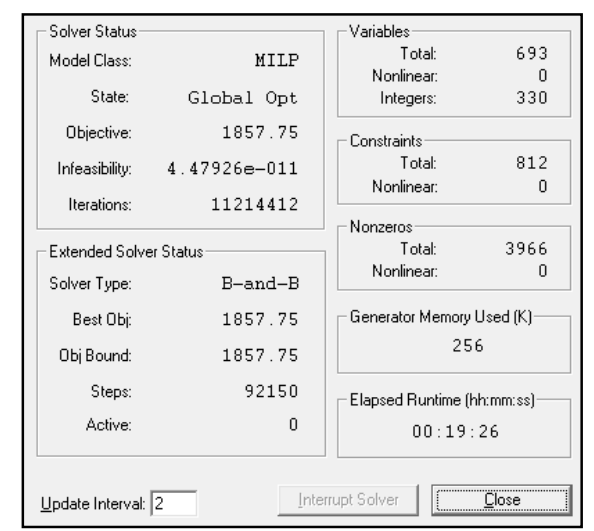

Fig. 1 Schematic diagram of the lingo solver

According to the result of the optimal solution, a schematic diagram of the optimized scheduling scheme is shown in Fig.2. The optimization comprehensively considers the minimum total travel distance of the vehicle and the minimum total ton-kilometer number, avoiding the long-distance cargo driving as much as possible, and making a full use of the loading capacity of each vehicle. The scheme is optimizing total vehicle travel distance by $49 \%$ over the original dispatch plan (each customer is serviced by only one car) significantly reducing costs. 


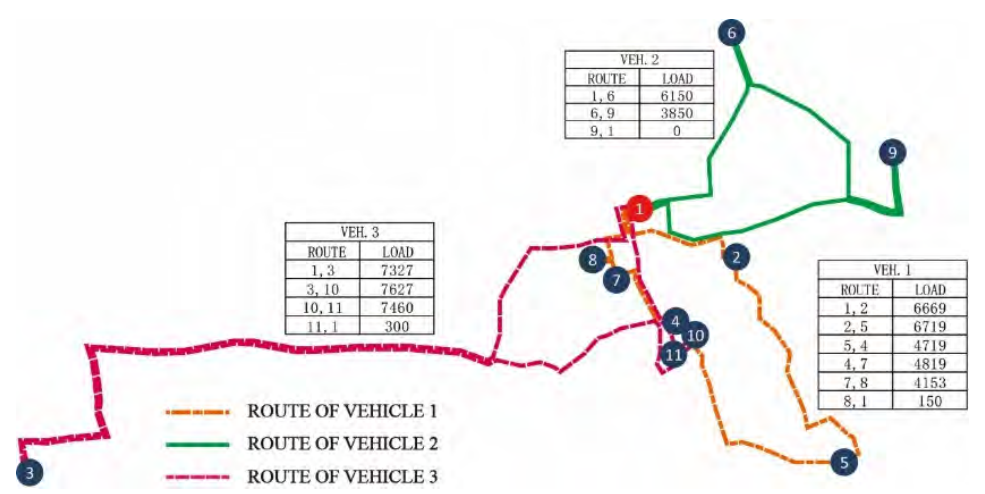

Fig. 2 Schematic diagram of optimized scheduling

\section{Acknowledgments}

Foundation items: National Key R\&D Program of China(2016YFE0201700).

\section{References}

[1]. Yang Ge. Coking Industry Path Optimization and Customer Service Impact_-Based on Ant Colony Algorithm[J]. Logistics Engineering and Management, 2013, 09: 82-83. M. P. Brown and K. Austin, Appl. Phys. Letters 85, 2503-2504 (2004).

[2]. Ding Jie. Research on Express Delivery Strategy Based on Depth First Search Algorithm[J]. Market Weekly: Theoretical Research, 2012(3); 21-23.

[3]. Shi Chunyan. Research on multi-vehicle vehicle routing problem with vehicle time window [D]. Chongqing Technology and Business University, 2015. 